\title{
MicroRNAs in Liver Health and Disease
}

\author{
Shu-hao Hsu • Kalpana Ghoshal
}

Published online: 13 January 2013

(c) Springer Science+Business Media New York 2013

\begin{abstract}
MicroRNAs (miRNAs), a class of short noncoding RNAs, have been studied intensely and extensively in the past decade in every aspect of biological processes, including cell differentiation, proliferation and death. These findings pointed out the pivotal role of miRNA in posttranscriptional control of gene expression in animals and established miRNAs as therapeutic targets for different pathophysiological processes, including liver disease. Here we have discussed the recent advances made in identifying the miRNAs deregulated in different liver diseases such as obesity, hepatitis, alcoholic and nonalcoholic steatohepatitis, cirrhosis and hepatocellular carcinoma, as well as pathophysiological conditions such as developmental abnormality. We have specifically reviewed the role of miRNAs in these diseases and discussed critically potential impacts of these miRNAs as biomarkers and/or therapeutic targets in liver pathobiology in the clinical setting. Finally, we have highlighted the latest techniques or preclinical and/or clinical trials that are being developed to replenish or inhibit the deregulated miRNAs.
\end{abstract}

\section{S. Hsu}

Department of Molecular and Cellular Biochemistry,

The Ohio State University, Columbus, OH 43210, USA

S. Hsu $(\bowtie) \cdot$ K. Ghoshal

Comprehensive Cancer Center, The Ohio State University,

420 West 12th Avenue, 606 TMRF Building, Columbus,

OH 43210, USA

e-mail: cothuho@gmail.com

K. Ghoshal

Department of Pathology, The Ohio State University,

Columbus, OH 43210, USA
Keywords microRNA - miRNA - Liver development . Metabolism - Hepatitis, Fibrosis · Hepatocellular carcinoma $\cdot$ HCC $\cdot$ CC $\cdot$ Liver disease - Gene therapy · Pathobiology

\section{Introduction}

MicroRNAs (miRNAs) are short noncoding RNAs consisting of about 22 nucleotides that negatively regulate expression of protein-coding genes. Although miRNAs are predominantly transcribed into primary miRNAs (primiRNA) by RNA polymerase II, a few are transcribed by RNA polymerase III. The mechanism of transcriptional regulation of miRNAs is not fully determined, because identification of the miRNA promoters is still a challenging task [1-3]. The pri-miRNA is processed by Drosha, a RNAse III ribonuclease, and its partner DGCR8 $[4,5]$ to generate pre-miRNA, which is then transported from the nucleus to cytoplasm by Exportin-5 [6]. In cytoplasm, Dicer, also an RNAse III endonuclease, interacts with TRBP (Tar RNA binding protein) to mediate further processing of pre-miRNA to a $\sim 22$ nucleotide miRNA duplex [7]. The single-stranded mature miRNA is then incorporated into effector complexes known as miRISC (miRNAinduced silencing complex) to suppress gene expression [8]. Generally, miRNAs regulate gene expression in animal cells by inhibiting translation and/or inducing decay of target mRNAs [9]. The deregulated miRNAs may lead to abnormal expression of their target genes and finally lead to diseases. Here, the discussion is focused on the deregulation of miRNAs in liver diseases (Fig. 1) and the potential therapeutic strategies developed from these findings. 


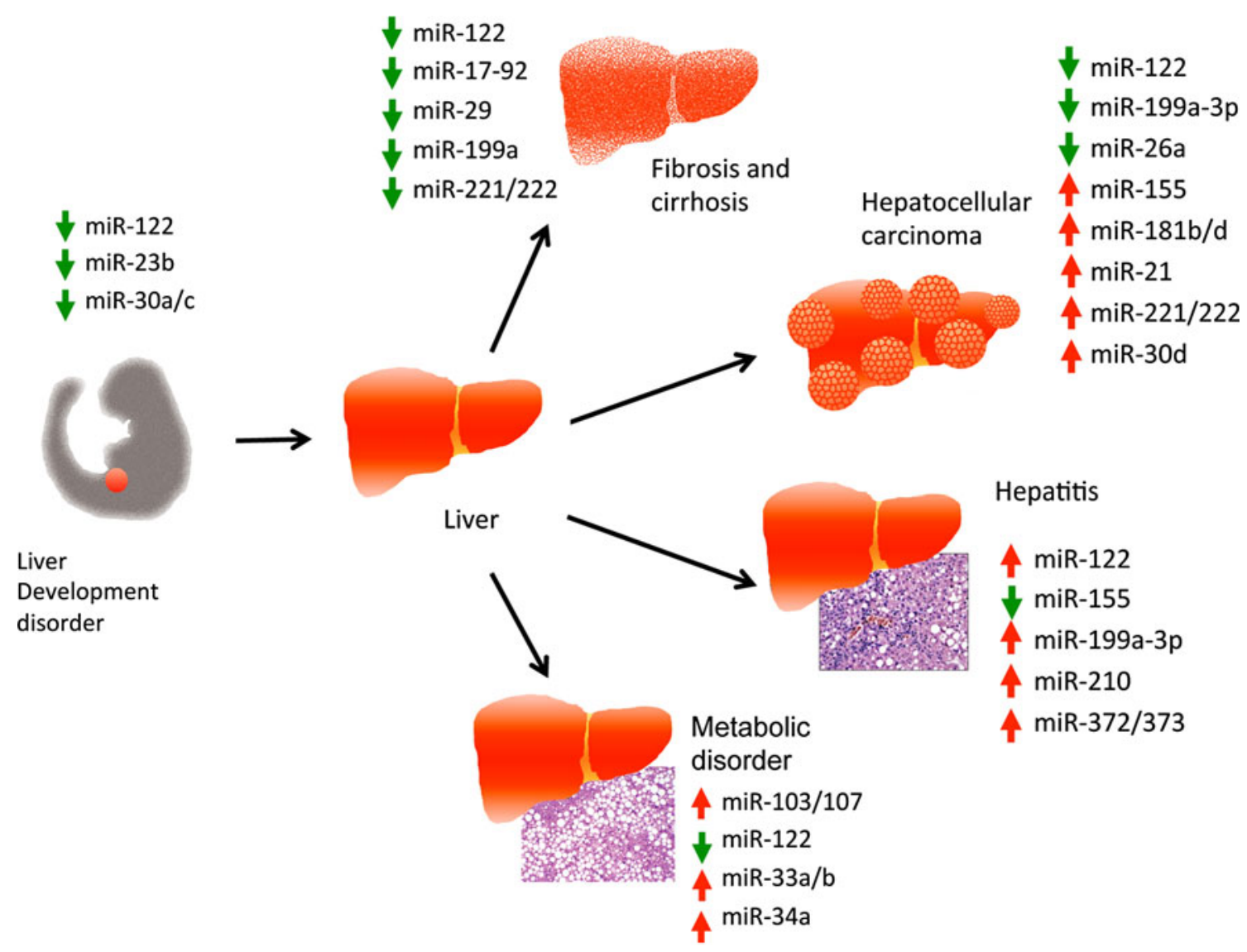

Fig. 1 Summary of deregulated miRNAs identified in different liver disease. The arrow indicates the abnormal expression pattern of each miRNA in the designated disease. Red, upregulated; Green, downregulated. (Color figure online)

\section{Role of miRNAs in Liver Development}

miRNAs are known to be important in the regulation of organ development and cell differentiation. For example, lin-4 and let-7, two of the founding members of miRNAs identified in C. elegans [10], were critical components to regulate different stages of development in C. elegans. Loss-of-function mutations in lin-4 and let-7 genes lead to delayed development at early larval and late-larval/adult stages, respectively, whereas increased expression of these miRs accelerates development of the organism. Similarly, the early embryonic lethality of Dicer 1 knockout mice confirmed its essential role in mammalian development [11]. However, its role in liver development has not been addressed because two studies that have reported phenotype of liver-specific Dicer 1 knockout mice used Alb-Cre mice where the gene is deleted after the development of the liver bud [12•, 13]. Crossing Dicer 1 floxed mice to AfpCre or Foxa3-Cre may be helpful to address the role of miRNAs in liver development. Apparently normal liver morphology in mice with deleted miR-122, the most abundant, highly conserved, liver-specific miRNA, demonstrated that miR-122 is not essential for embryonic liver development $[14 \bullet \bullet, 15 \bullet \cdot$. However, it is likely that there are functional redundancies among liver-specific miRs complementing the function of miR-122 during development, which can be addressed by deleting several fetal liver-specific miRNA genes in combination in mice.

Recent studies from several labs, including ours, have established essential role of miR-122 in maintaining the differentiation state of the liver, since its loss induced proliferation of oval and bile duct cells in young adult mice and hepatocellular carcinoma HCC with age [14••]. In addition to miR-122, several other miRNAs appear to be involved in liver differentiation. Antisense oligo (ASO) mediated knockdown of miR-30a, specifically expressed in the ductal plate and the bile duct, results in defects in the intrahepatic bile duct and canaliculi in zebrafish [16]. Furthermore, the miR-23b cluster downregulates TGF- $\beta$ / BMP signaling, which governs differentiation of hepatocytes and cholangiocytes $[17,18]$ by silencing Smad 3, 4 and 5 [19]. It is proposed that the gradient expression of miR-23b clusters negatively regulate TGF- $\beta$ expression and promote terminal differentiation of bi-potential hepatoblasts to hepatocytes instead of bile duct cells. Many studies have revealed differential expression of miRNAs 
between embryonic and adult livers and correlated their findings with the critical factors involved in liver development. However, the expression of miRNA often observed in the total liver RNA may not reflect the actual biological role of differentially expressed miRNA in specific cell types. Therefore, it is necessary to analyze the miRNA expression at different stages of liver development by monitoring their expression in specific cell types by in situ hybridization or real-time RT-PCR in a purified cell population. Also, in absence of knockout animal models, it is difficult to pinpoint the developmental role of these miRs.

\section{miRNAs in Obesity and its Associated Complications}

Partly due to changed dietary habits of excessive high caloric intake and inactive lifestyles [20], obesity has become a major health problem by causing cardiovascular disorders such as atherosclerosis [21] or metabolic disorders such as type II diabetes [22]. Obesity is mainly induced by excessive storage of energy in adipose tissue and a subsequent increase in the size and number of adipocytes. Excessive production and storage of energy are frequently associated with abnormal lipid metabolism in the liver resulting from miRNA deregulation. Among the deregulated miRNA in obese mouse model, miR-34a has been reported to be the most highly elevated hepatic miR. Antisense mediated miR-34a inhibition restored hepatic expression of $\beta$-Klotho, a critical enzyme in bile acid/ cholesterol metabolism [23], and improved FGF19 signaling pathway that mediated postprandial response and decreased liver fat [24]. FGF19 signaling is frequently impaired in patients with liver steatosis. Overexpression of let-7a in the pancreas leads to decreased fat mass, body weight and size through enhanced glucose tolerance. Knockdown of let-7 by antisense oligonucleotides prevents obesity-induced glucose intolerance and steatosis [25]. The control of let-7a over glucose homeostasis is partly through targeting the insulin receptor (Insr) and insulin receptor substrate 2 (Irs2). Similarly, silencing of miR-103/107, both abnormally upregulated in an obese mouse model, leads to insulin sensitivity and improves glucose homeostasis [26]. This effect appears to be through its direct target, caveolin-1, which is an important regulator of insulin receptors [26].

miRNAs that regulate lipid metabolism by targeting several key genes have been extensively studied [27]. MiR122 has been one of the most widely studied miRNAs that affects lipid metabolism [28, 29]. Esau et al. first showed that the application of a modified antisense oligonucleotide (ASO) against miR-122 efficiently downregulated serum cholesterol in high-fat diet fed mice [28]. ASO-mediated depletion of miR-122 activated AMPK and led to elevated fatty acid oxidation and inhibition of cholesterol synthesis in the liver. AMPK is known to be a master regulator of metabolism in different organs, including muscle, brain and liver [30], and has been considered as a therapeutic target for obesity-induced type II diabetes. The anti-diabetic drug metformin functions by activating AMPK signaling pathway, leads to reduced glucose synthesis in liver and ameliorates insulin resistance in muscle. In the light of this study by Esau et al. on the effect of miR-122 on AMPK, miRNAs can also be considered as an option to treat obesity-induced disease through AMPK activation in the liver. Recently, miR-199a-3p, miR-195, and miR-451 were shown to target upstream kinase activating AMPK, including LKB1 [31] in hepatocytes and MO25 in glioma cells [32].

On the other hand, genes involved in lipid metabolism may function by regulating miRNA expression. For example, it has been shown that activation of SREBP1 and SREBP2, which modulate cholesterol homeostasis by activating sterol-regulated genes, induce transcription of miR-33a and miR-33b, located within the intronic region of SREBP2 and SREBP1, respectively [33•]. MiR-33a/b were shown to target ABCA1, involved in cholesterol efflux. Inhibition of miR-33 by ASO further reversed the cholesterol efflux to prevent atherosclerosis in mice [34.•] and raised plasma HDL-associated cholesterol (HDL-C) and VLDL-associated triglyceride in non-human primates [35]. These findings further highlighted the therapeutic potential of miRNAs in the treatment of athesclerosis by regulating HDL-C. It is clinically feasible to target several key miRNAs simultaneously to control the activity of key regulators in lipid metabolism, such as AMPK, which is an important drugable target for pharmaceutical companies. However, most of the research has been focused on the effect of single miRNAs on one aspect of metabolism, and it is too early to speculate on the outcome of treatment targeting multiple miRNAs. Thus, it is necessary to establish a "miRNAomic" analysis in patients with metabolic disorders in order to fully understand which group of miRNAs are the most critical targets associated with the designated diseases. Furthermore, from the intriguing study made by Gatfield et al. [36•] on the role of miR-122 in hepatic circadian gene expression, we should be aware that temporal expression pattern of miRNAs may be key to the fine tuning of metabolism and can be used to accurately detect or prevent metabolic disorder.

\section{Role of miRs in Viral Hepatitis}

Hepatitis is predominantly caused by hepatitis B virus (HBV) and hepatitis $\mathrm{C}$ virus (HCV) infection. Although there are effective therapies to inhibit viral replication, 
none are curative because viruses often develop resistance to these drugs. Several important findings on the implication of miR-122 in $\mathrm{HCV}$ replication highlighted the potential of miRNAs in the development of anti-viral therapy. The seminal discovery by Jopling et al. [37] demonstrating essential role of miR-122 in the replication of HCV RNA in hepatic cells was instrumental in the development of Miravirsen, a modified anti-miR-122 oligonucleotide by Santaris Pharma, currently undergoing phase II clinical trials in HCV-infected patients. MiR-122 binds to two cognate sites located in the proximal $5^{\prime}$-end of the viral genome to protect it from $5^{\prime}$ to $3^{\prime}$ exonuclease activity or cytoplasmic sensors of viral RNA, and subsequently to facilitate replication [37]. Mutation of miR-122 binding sites on HCV $5^{\prime} \mathrm{UTR}$ significantly inhibits HCV viral RNA loading, which can be restored by the overexpression of miR-122 oligonucleotides containing compensatory mutated $3^{\prime} U T R$. This specific interaction between miR-122 and HCV makes miR-122 an ideal target for the development of anti-HCV therapy. Liver-specific delivery of Miravirsen, a locked nucleic acid phosphorthioate modified antisense oligonucleotide (LNA-ASO), was developed to effectively targets miR-122 in hepatocytes after systemic delivery [38••, 39].

In contrast to $\mathrm{HCV}$, much less progress has been made on the development of miRNA based anti-HBV therapy. To identify miRs critical for HBV replication, 328 human miRNAs were individually knocked-down in HepG2.2.15 cells that are genetically designed to support replication of HBV genome. Among these, loss of miR-199a-3p and miR-210 reduced viral replication by targeting HBV $\mathrm{S}$ protein (HBsAg) and $\mathrm{HBV}$ e antigen ( $\mathrm{HBeAg}$ ), respectively. Further, certain miRNAs indirectly regulate several critical host proteins involved in HBV replication. Cyclin G1 was previously identified as a miR-122 target, and it regulates HBV replication by blocking p53-mediated inhibition of HBV transcription [40]. MiR-372/373 was shown to promote HBV expression in HepG2.2.15 cells by targeting the transcription factor NFIB [41]. Our previous study also reported that miR-155 negatively regulated CCAAT/enhancer binding protein beta $(\mathrm{C} / \mathrm{EBP} \beta)$, which activates $\mathrm{HBV}$ transcription by binging to its core and $\mathrm{S}$ promoters [42, 43]. Moreover, miRNA may regulate HBV replication indirectly. For example, ectopic expression of miR-1 resulted in marked increase of HBV replication by inducing HBV transcription through indirect augmentation of farnesoid $X$ receptor expression [44]. These findings shed further light on the development of miR-based HBV therapy. However, the majority of the studies on the mechanism of HBV were based on human HCC cell lines due to limited availabilities of animal models that can be naturally infected by HBV $[45,46]$. Therefore, it will be necessary to perform these studies in primates or animal models with humanized livers [47] to further extend and confirm these studies in vivo.

\section{Role of miRs in Liver Fibrosis}

Liver fibrosis and subsequent cirrhosis usually predispose humans to liver cancer or liver failure due to the pro-tumorigenic microenvironment caused by fibrotic tissues surrounding hepatocytes [48]. Therefore, therapeutic strategies targeting fibrosis are critical for the prevention of cirrhosis and the transformation of normal hepatocytes into cancer cells.

When the liver is exposed to chronic damage or inflammation, fibrosis develops through the excessive accumulation of extracellular matrix (ECM) secreted by hepatic stellate cells (HSC). Numerous studies have suggested that miRNAs play a critical role in the activation of HSC and the secretion of ECM. Recent studies from our and other laboratories have shown that an increased occurrence of fibrosis accompanied by steatohepatitis was observed on miR-122 knockout mice [14••, 15••]. The onset of hepatic fibrosis after miR-122 depletion may be partially due to the increased expression of Klf6, which is a zinc finger transcription factor and a tumor suppressor [15•*]. However, reduction of Klf6 was also shown to promote fibrosis in a rodent chronic liver injury model [49].

The expression of miRNAs involved in fibrosis was shown to be regulated by several genes. In liver specific Mdm2 deleted mice, Mdm2-mediated p53 degradation was impaired and resulted in spontaneous fibrosis through repressing miR-17-92, which targets connective tissue growth factor, a master regulator of fibrosis [50]. In hepatic stellate cells, miR-29 was downregulated by transforming growth factor beta (Tgf- $\beta$ ) and inflammation. The reduced miR-29 expression resulted in higher expression of collagen genes, including Collal, Col4a5, and Col5a3 [51]. Similar observation was made in pulmonary fibrosis [52]. Furthermore, enhanced fibrosis and mortality were reported in hepatocyte-specific miR-29 knockout mice in response to carbon tetrachloride, and altered expression of genes implicated in fibrosis such as $P d g f$ [53]. These findings suggested knockdown of miR-29 via ASO could be potential new therapeutic target for fibrosis.

Another causal factor in hepatic fibrosis is inflammation. HSC can be activated by inflammatory cytokines such as TNF- $\alpha$, IL-6, IL-1, and CC-chemokine ligand 2 (CCL2) [54]. Interestingly, our studies have shown that hepatic CCL2 is a direct target of miR-122 and upregulated in miR-122 knockout mice, which is responsible for the hepatic recruitment of $\mathrm{CD} 11 \mathrm{~b}^{\text {high }} \mathrm{Gr}-1^{+}$neutrophil/monocytes known to cause inflammation by secreting pro-inflammatory

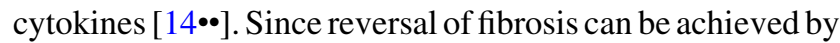
switching activated HSCs to quiescent status [55], it should 
be feasible to reverse fibrosis by the delivery of miRNAs, such as miR-122, targeting cytokines/chemokines that activate HSCs. Furthermore, miRNAs could be a diagnostic biomarker for liver fibrosis. For example, miR-199a-5p/ $199 a-3 p$ and miR-221/222 were found to be upregulated in fibrotic human liver and mouse models of liver fibrosis [56]. Interestingly, miR-222 expression in HSCs is transcriptionally upregulated by NF- $\kappa \mathrm{B}$ [57] and NF- $\kappa \mathrm{B}$ activator, TNF- $\alpha$ and TGF- $\alpha[56]$. NF- $\kappa$ B is a critical factor activating stellate cells in the process of fibrogenesis [58, 59]. These studies suggested that miR-221/222 is a good biomarker for activated HSCs and fibrosis. From all these evidence, it is apparent that miRNAs are likely to be promising therapeutic targets since they are critical regulators of HSC activation.

\section{miRNAs as Biomarkers and Therapeutic Targets in $\mathrm{HCC}$}

Carlo Croce's group was the first to connect miRNAs to cancer by means of a large number of studies in the miRNA field, which are focused on identifying miR signature associated with different cancers, because of miRNA profiling is more reproducible than mRNA profiling because of its higher stability, and because it can be done with paraffin-embedded tissue sections.

Like mRNAs, miRs can also function as oncogenes or tumor suppressors depending upon the cellular context. Development of spontaneous tumors induced by deletion of a single miRNA, miR-122, in the liver indicates that this miRNA indeed functions as a tumor suppressor [14••, 15••]. MiR122 knockout mice independently developed by us and by Ann-Ping Tsou's group, are the first HCC model organism based on miRNA deletion. MiR-122, the most abundant miRNA in liver, was first suggested as a tumor suppressor in liver due to its diminished expression in primary HCCs of human and rodent origin, and its ectopic expression studies in HCC cell lines and xenograft models [60, 61]. Loss of miR122 results in the upregulation of its target genes, including Adam10/17, IgflR, Srf, and Ccngl [61], and inhibits tumorigenic properties of HCC cells. In addition, miR-199a-3p, also abundantly expressed in the liver, was significantly suppressed in $40 \mathrm{HBV}$-related HCC specimens [62]. Similarly, miR-199a-3p deficiency induces ERK signaling pathway partly through targeting PAK4. Moreover, the miRNAs analysis of a large cohort of HCC patients with radical tumor resection revealed that $\mathrm{miR}-26 \mathrm{a} / \mathrm{b}$ were significantly suppressed in tumors compared to noncancerous tissues [63]. Ji et al. [63] proposed that the miR-26a deficiency may promote tumorigenesis by the activation of NF- $\kappa$ B and IL- 6 signaling pathways. Dramatic tumor suppression by the adeno-associated viral delivery of miR-26a in a Myc driven mouse model of HCC further suggests that miR-26a may inhibit tumor growth by directly targeting genes involved in the control of cell cycle, such as $C c n d 2$ and $C c n e 2[64 \bullet \cdot]$.

Similar to the studies with tumor suppressor miRNAs, many studies were conducted to discover the oncogenic miRNAs. MiR-21, which is widely upregulated in different types of cancers, was upregulated in human HCC and may promote tumor growth by targeting phosphatase and tensin homolog (PTEN). PTEN is an important suppressor that is involved in suppression of cell migration and invasion by inhibiting its downstream targets including $F A K, M M P 2$ and $M M P 9$ [65]. Furthermore, in a choline-deficient and amino-acid-defined (CDAA) diet-induced HCC model, many oncogenic miRNAs, including miR-155, miR-221/ 222 , miR-21 and miR-181b/d, were upregulated at an early stage of hepatocarcinogenesis [66-69]. Interestingly, these miRs were reported to target tissue inhibitor of metalloprotease 3 (TIMP3) [67-69], which is involved in tumor invasion and metastasis as PTEN. Notably, miR-122 was not associated with any region with frequent chromosomal loss, which suggests the loss of miR-122 expression in HCC may not be due to chromosomal aberrations. Taken these findings together, it is clear that the deregulation of miRNAs contributes to the hepatocarcinogenesis. However, it is necessary to generate animal models ectopically expressing or deplete of these deregulated miRNA, since many of their proposed functions were concluded based on in vitro studies in HCC cell lines. Without direct evidence demonstrating that these aberrantly expressed miRNAs are causally linked to HCC development in vivo, it would be premature to translate these findings into HCC therapy.

\section{MicroRNA Therapeutics for Liver Disease}

With the advent of onco- and tumor suppressor-miRs, much interest has been focused on taking microRNAs from the bench to the bedside. The advantages of miR-based therapies are their smaller size and their ability to regulate a large number of cellular targets. Different strategies to deliver miRmimics or inhibitors are being explored to restore gene deregulation caused by the loss- or gain-of-function of miRNAs, respectively. The stabilization of delivered oligonucleotides in vivo was the first critical hurdle to overcome. Various vehicles or chemical modifications were designed and tested from cells in culture, experimental animal models and recently in clinical applications [70]. Table 1 summarizes the important findings on the development of these techniques in the context of liver-targeted delivery of miRNAs.

\section{Viral Delivery of miRNAs in Liver Disease}

Generally, the delivery methods of miRNAs can be classified into viral and non-viral systems. The advantage of 
Table 1 Summary of selected studies on the in vivo delivery of miRNA mimics or inhibitors

\begin{tabular}{|c|c|c|c|c|c|}
\hline Delivery media & Target miR & Targeted disease & Animal model & $\begin{array}{l}\text { Methods of } \\
\text { administration }\end{array}$ & Ref \\
\hline $\begin{array}{l}\text { Adeno-associated virus/ } \\
\text { miRNA }\end{array}$ & $\operatorname{miR}-26$ & $\mathrm{HCC}$ & Myc-induced HCC & Intravenous & {$[64 \bullet \bullet]$} \\
\hline $2^{\prime}$-F/MOE modified antimir & miR-33 & Atherosclerosis & Ldlr-/- mice & Subcutaneous & {$[34 \bullet \bullet]$} \\
\hline LNA-modified antimir & miR-33 & Atherosclerosis & Western-diet fed mice & Intravenous & {$\left[33^{\bullet}\right]$} \\
\hline $\begin{array}{l}\text { Lipid Nanoparticles/ } \\
\text { antagomirs }\end{array}$ & $\begin{array}{l}\mathrm{miR}-103 \\
107\end{array}$ & $\begin{array}{l}\text { Type } 2 \text { diabetes } \\
\text { obesity }\end{array}$ & Chow-fed mice & Intravenous & {$[98 \bullet \bullet]$} \\
\hline LNA-modified antagomir & $\operatorname{miR}-122$ & Iron homeostasis & Chow-fed mice & Intraperitoneal & [99] \\
\hline $\begin{array}{l}\text { Adeno-associated virus/ } \\
\text { miRNA }\end{array}$ & miR-122 & $\mathrm{HCC}$ & Myc-induced HCC & Intravenous & {$[14 \bullet \bullet]$} \\
\hline $2^{\prime}$-O-Me antagomir & $\operatorname{miR}-122$ & $\begin{array}{l}\text { Cholesterol } \\
\text { homeostasis }\end{array}$ & Chow-fed mice & Intravenous & [29] \\
\hline $2^{\prime}$-MOE modified antimir & $\operatorname{miR}-122$ & Obesity & Diet-induced obesity mice & Intraperitoneal & [28] \\
\hline LNA modified antimir & miR-122 & Hypercholesteroemia & $\begin{array}{l}\text { Chow-fed mice/African green } \\
\text { monkeys }\end{array}$ & $\begin{array}{l}\text { Intravenous/ } \\
\text { Intraperitoneal }\end{array}$ & {$[38 \bullet \bullet]$} \\
\hline LNA modified anti-mir & miR-122 & $\mathrm{HCV}$ & Chimpanzee & Intravenous & {$[93 \bullet \bullet]$} \\
\hline $\begin{array}{l}\text { Lipid nanoparticle/miR } \\
\text { mimic }\end{array}$ & miR-124 & $\mathrm{HCC}$ & DEN-induced HCC & Intravenous & {$[74]$} \\
\hline
\end{tabular}

viral delivery is that a single administration of recombinant viral particles yields the long-term expression of specific miRNAs of interest, and the infected cells need not be replication proficient. The viral vector is constructed with genes encoding the hairpin structure of miRNA under the control of a promoter activated by RNA polymerase II. Adeno-associated virus (AAV) is successfully used as the vehicle to deliver miRNA to liver through systemic circulation [64•]. AAV simultaneously infects dividing and non-diving cells with an infrequent or site-specific integration of its genes into host's genome [71]. As mentioned above, Kota et al. [64•] and Hsu et al. [14•] demonstrated the AAV-mediated delivery of miR-26a and miR-122 precursor genes respectively to Myc-induced HCC model successfully inhibited tumor growth. Similar approaches can be applied to different types of cancers. For example, Trang et al. [72] showed that adenoviral delivery of let-7a through respiratory inhalation suppressed the tumor growth to a murine lung tumor model.

\section{Non-Viral Delivery of miRNAs in Liver Disease}

Although viral delivery of miRNAs produces significant ectopic expression of target genes, non-viral systems are still considered a safer choice to prevent many adverse effects of viruses, including immune system stimulation and the risk of inducing oncogenic transformation [73]. Among the numerous chemical vehicles for the non-viral delivery of RNAi, cationic lipid-based nanoparticles (cLNPs) are widely used for therapy targeting the liver
[74-77, 78•, 79] and other organs [80-84]. Compared to a neutral lipid system, the positively charged cLNPs can easily interact with negatively charged miRNAs [85] to form lipoplexes that prevent miRNAs from RNAse degradation in blood circulation, facilitate incorporation of anionic miRNAs into anionic cell membranes, and promote disruption of endosomal membrane to release engulfed miRNAs [86, 87]. An important advance of liver-targeted siRNA/miRNA delivery is the development of stable nucleic-acid lipid particles (SNALP), which are natural cationic lipid nanoparticles preferably taken up by the liver. Upon reaching the liver, the SNALP could exit the intravascular space to directly access hepatocytes as long as the particle size is smaller than the pore size of the fenestrated vasculature (100-150 nm in diameter) [88]. Zimmermann et al. [77] have used SNALP-mediated delivery of siRNA to target hepatic APOB in non-human primates. Notably, with a single intravenously injection APOB in liver was reduced by $90 \%$, and the effect of downregulation lasted for 11 days without causing significant tissue toxicity. SNALP has been tested in clinical trials and may be used in clinic in the near future [89].

\section{Delivery of miRNA Inhibitors}

Compared to chemical-vehicle-mediated delivery of miR mimics, delivery of miR inhibitors depends more on the chemical modification of the inhibitors. Krutzfeldt et al. [29] first demonstrated miR-122 knockdown in vivo by the systemic delivery of an antagomirs, which is a 
2'-O-methyl-, phosphorothioate- modified, and cholesterol conjugated oligoribonucleotides complementary to specific microRNAs. This modification was also shown to effectively deliver anti-miR-122 to liver and suppress the proliferation of orthotopically xenografted tumor cells in mouse livers [90]. The 2'-O-methyl modification was further improved by locked nucleic acid (LNA) chemistry, in which there is an extra bridge connecting the $2^{\prime}$ oxygen and $4^{\prime}$ carbon of pentose, to render high binding affinity to complementary RNA target molecules and high stability in blood and tissues in vivo [91]. With LNA modification, the length of antimir was shortened significantly to cover only the seed sequence of selected miRNAs, resulting in much higher affinity compared to the full-length antimirs [38••, 39]. LNA-modified antimirs have several other advantages, including low off-target effects [92], efficient suppression of entire families of miRNA [39], and broad distribution throughout different organs after systemic delivery. The application of this LNAASO was first tested in chimpanzees chronically infected with $\mathrm{HCV}$, which load titer without occurrence of mutated virus escaped from treatment [93॰]. However, our own and other recent studies $[14 \bullet \bullet, 15 \bullet \cdot]$ have highlighted the concern that long-term inhibition of miR-122 may lead to the development of hepatitis and liver cancer. Our data showed that miR-122 liver-specific and germ line knockout mice exhibited high incidences of HCC. According to our unpublished data, about $15 \%$ of heterozygous miR-122 knockout mice, which had $50 \%$ reduction of miR-122 level, developed HCC after a longer time period ( $\sim 20$ months) than complete knockout mice ( $\sim 12$ months). Therefore, HCV-infected patients undergoing Miravirsen therapy should be routinely monitored for liver cancer development.

\section{Conclusions and Future Directions}

Gene therapy has been proposed and applied in some cases to restore normal gene expression in liver disease. For instance, gene therapy was proposed to treat HCC patients without surgical removal of the tumor mass [94-96]. Although siRNA-mediated silencing of the expression of a specific disease-causing target gene holds great promise for gene therapy, it is likely that redundant genes with complementary functions could compensate for the function of the knockdown gene. As an alternative strategy, miR-based therapy is being intensely pursued because miRNAs can target multiple genes. The fast growing discoveries of miRNAs involved in liver physiology and pathophysiology will greatly strengthen the therapeutic potential of gene delivery based on miRNAs in treatment for liver disease.

Most of the studies relating miRs to a specific disease are based on identifying and validating a few candidate targets predicted by different databases. However, in the absence of an unbiased transcriptomic and/or proteomic approach to identify biological targets of miRNAs in specific cell types/ tissues, it is difficult to evaluate the molecular functions of specific miRs in different diseases. Therefore, before embarking on expensive clinical trials, it will be important to perform bioinformatics-based analysis in a large number of patients with different liver disease. The database of disease-associated miRNA signatures will help to select the best treatment option for patients with different patterns of gene deregulation. For example, HCC patients with lowmiR-122 expression, as shown in many studies [14, 62, 97], are more suitable for miR-122 delivery than patients with high-miR-122 expression. In other words, the miR-122 delivery should not be a prioritized option for the treatment of HCCs expressing a high level of miR-122.

Disclosure S. Hsu is supported by National Institutes of Health (NIH) Grants to K. Ghoshal. K. Ghoshal is supported by Grants from $\mathrm{NIH}$, and has received honoraria for speaking engagements from FASEB and University of Colorado in Denver.

\section{References}

Papers of particular interest, published recently, have been highlighted as:

- Of importance

•• Of major importance

1. Lee $Y$, Kim M, Han J et al (2004) MicroRNA genes are transcribed by RNA polymerase II. EMBO J 23(20):4051-4060

2. Cai X, Hagedorn CH, Cullen BR (2004) Human microRNAs are processed from capped, polyadenylated transcripts that can also function as mRNAs. RNA 10(12):1957-1966

3. Bracht J, Hunter S, Eachus R et al (2004) Trans-splicing and polyadenylation of let-7 microRNA primary transcripts. RNA 10(10):1586-1594

4. Han J, Lee Y, Yeom KH et al (2004) The Drosha-DGCR8 complex in primary microRNA processing. Genes Dev 18(24): 3016-3027

5. Lee Y, Ahn C, Han J et al (2003) The nuclear RNase III Drosha initiates microRNA processing. Nature 425(6956):415-419

6. Lund E, Guttinger S, Calado A et al (2004) Nuclear export of microRNA precursors. Science 303(5654):95-98

7. Haase AD, Jaskiewicz L, Zhang $\mathrm{H}$ et al (2005) TRBP, a regulator of cellular PKR and HIV-1 virus expression, interacts with Dicer and functions in RNA silencing. EMBO Rep 6(10):961-967

8. Lingel A, Sattler M (2005) Novel modes of protein-RNA recognition in the RNAi pathway. Curr Opin Struct Biol 15(1): $107-115$

9. Winter J, Jung S, Keller S et al (2009) Many roads to maturity: microRNA biogenesis pathways and their regulation. Nat Cell Biol 11(3):228-234

10. Reinhart BJ, Slack FJ, Basson M et al (2000) The 21-nucleotide let-7 RNA regulates developmental timing in Caenorhabditis elegans. Nature 403(6772):901-906

11. Bernstein E, Kim SY, Carmell MA et al (2003) Dicer is essential for mouse development. Nat Genet 35(3):215-217 
12. - Sekine S, Ogawa R, Ito R et al: Disruption of Dicer1 induces dysregulated fetal gene expression and promotes hepatocarcinogenesis. Gastroenterology 2009, 136(7):2304-2315 e2301-2304. This paper demonstrated loss of expression of miRs by the liver specific deletion of Dicer1 directly promote hepatocarcinogenesis.

13. Hand NJ, Master ZR, Le Lay J, Friedman JR (2009) Hepatic function is preserved in the absence of mature microRNAs. Hepatology 49(2):618-626

14. •• Hsu SH, Wang B, Kota J et al (2012) Essential metabolic, antiinflammatory, and anti-tumorigenic functions of miR-122 in liver. J Clin Investig. Together with ref\#15, this study showed that the liver specific or germline deletion of miR-122 directly resulted in spontaneous HCC in mice. The deletion of miR-122 also induced steatohepatitis through the upregulation of target genes, Agpat1 and Ccl2, respectively.

15. • Tsai WC, Hsu SD, Hsu CS et al (2012) MicroRNA-122 plays a critical role in liver homeostasis and hepatocarcinogenesis. J Clin Investig. Together with ref\#15, this study showed that the liver specific or germline deletion of miR-122 directly resulted in spontaneous HCC in mice. The deletion of miR-122 also induced steatohepatitis through the upregulation of target genes, Agpat1 and $\mathrm{Ccl} 2$, respectively.

16. Hand NJ, Master ZR, Eauclaire SF et al (2009) The microRNA30 family is required for vertebrate hepatobiliary development. Gastroenterology 136(3):1081-1090

17. Clotman F, Jacquemin P, Plumb-Rudewiez N et al (2005) Control of liver cell fate decision by a gradient of TGF beta signaling modulated by Onecut transcription factors. Genes Dev 19(16): 1849-1854

18. Grasl-Kraupp B, Rossmanith W, Ruttkay-Nedecky B et al (1998) Levels of transforming growth factor beta and transforming growth factor beta receptors in rat liver during growth, regression by apoptosis and neoplasia. Hepatology 28(3):717-726

19. Rogler CE, Levoci L, Ader T et al (2009) MicroRNA-23b cluster microRNAs regulate transforming growth factor-beta/bone morphogenetic protein signaling and liver stem cell differentiation by targeting Smads. Hepatology 50(2):575-584

20. Popkin BM, Doak CM (1998) The obesity epidemic is a worldwide phenomenon. Nutr Rev 56(4 Pt 1):106-114

21. McGill HC Jr, McMahan CA, Herderick EE et al (2002) Obesity accelerates the progression of coronary atherosclerosis in young men. Circulation 105(23):2712-2718

22. Kahn SE, Hull RL, Utzschneider KM (2006) Mechanisms linking obesity to insulin resistance and type 2 diabetes. Nature 444 (7121):840-846

23. Ito S, Fujimori T, Furuya A et al (2005) Impaired negative feedback suppression of bile acid synthesis in mice lacking betaKlotho. J Clin Investig 115(8):2202-2208

24. Fu T, Choi SE, Kim DH et al (2012) Aberrantly elevated microRNA-34a in obesity attenuates hepatic responses to FGF19 by targeting a membrane coreceptor beta-Klotho. Proc Natl Acad Sci USA 109(40):16137-16142

25. Frost RJ, Olson EN (2011) Control of glucose homeostasis and insulin sensitivity by the Let-7 family of microRNAs. Proc Natl Acad Sci USA 108(52):21075-21080

26. Yamamoto M, Toya Y, Schwencke C et al (1998) Caveolin is an activator of insulin receptor signaling. J Biological Chem 273 (41):26962-26968

27. Rottiers V, Naar AM (2012) MicroRNAs in metabolism and metabolic disorders. Nat Rev Mol Cell Biol 13(4):239-250

28. Esau C, Davis S, Murray SF et al (2006) miR-122 regulation of lipid metabolism revealed by in vivo antisense targeting. Cell Metab 3(2):87-98

29. Krutzfeldt J, Rajewsky N, Braich R et al (2005) Silencing of microRNAs in vivo with 'antagomirs'. Nature 438(7068): 685-689
30. Hardie DG (2003) Minireview: the AMP-activated protein kinase cascade: the key sensor of cellular energy status. Endocrinology 144(12):5179-5183

31. Lee CG, Kim YW, Kim EH et al (2012) Farnesoid X receptor protects hepatocytes from injury by repressing miR-199a-3p, which increases levels of LKB1. Gastroenterology 142(5):1206-1217 e1207

32. Godlewski J, Nowicki MO, Bronisz A et al (2010) MicroRNA451 regulates LKB1/AMPK signaling and allows adaptation to metabolic stress in glioma cells. Mol Cell 37(5):620-632

33. - Najafi-Shoushtari SH, Kristo F, Li Y et al (2010) MicroRNA-33 and the SREBP host genes cooperate to control cholesterol homeostasis. Science 328(5985):1566-1569. This paper revealed the role of miR-33 in the control of cholesterol homeostasis through targeting $A B C A 1$.

34. •• Rayner KJ, Sheedy FJ, Esau CC et al 2011 Antagonism of miR33 in mice promotes reverse cholesterol transport and regression of atherosclerosis. J Clinical Investig 121(7):2921-2931. First study to show the antimiR mediated miR-33 reduction reversed cholesterol transport and alleviate atherosclerosis in mouse model.

35. Rayner KJ, Esau CC, Hussain FN et al (2011) Inhibition of miR$33 \mathrm{a} / \mathrm{b}$ in non-human primates raises plasma HDL and lowers VLDL triglycerides. Nature 478(7369):404-407

36. • Gatfield D, Le Martelot G, Vejnar CE et al (2009) Integration of microRNA miR-122 in hepatic circadian gene expression. Genes Dev 23(11):1313-1326. This paper showed that miR-122 was tightly associated with control mechanisms regulating circadian gene expression.

37. Jopling CL, Yi M, Lancaster AM et al (2005) Modulation of hepatitis $\mathrm{C}$ virus RNA abundance by a liver-specific MicroRNA. Science 309(5740):1577-1581

38. •• Elmen J, Lindow M, Schutz S et al (2008) LNA-mediated microRNA silencing in non-human primates. Nature 452(7189): 896-899. This study demonstrated the therapeutic potential of LNA-antimiRs by evaluating miRNA function after systemic administeration of LNA-antimiRs in rodents and primates.

39. Obad S, dos Santos CO, Petri A et al (2011) Silencing of microRNA families by seed-targeting tiny LNAs. Nat Genet 43(4): 371-378

40. Wang S, Qiu L, Yan X et al (2012) Loss of microRNA 122 expression in patients with hepatitis B enhances hepatitis B virus replication through cyclin G(1) -modulated P53 activity. Hepatology 55(3):730-741

41. Guo H, Liu H, Mitchelson K et al (2011) MicroRNAs-372/373 promote the expression of hepatitis $\mathrm{B}$ virus through the targeting of nuclear factor I/B. Hepatology 54(3):808-819

42. Bock CT, Kubicka S, Manns MP, Trautwein C (1999) Two control elements in the hepatitis B virus S-promoter are important for full promoter activity mediated by CCAAT-binding factor. Hepatology 29(4):1236-1247

43. Lopez-Cabrera M, Letovsky J, Hu KQ, Siddiqui A (1990) Multiple liver-specific factors bind to the hepatitis B virus core/ pregenomic promoter: trans-activation and repression by CCAAT/ enhancer binding protein. Proc Natl Acad Sci USA 87(13): 5069-5073

44. Zhang $X$, Zhang E, Ma Z et al (2011) Modulation of hepatitis B virus replication and hepatocyte differentiation by MicroRNA-1. Hepatology 53(5):1476-1485

45. Menne S, Roneker CA, Korba BE et al (2002) Immunization with surface antigen vaccine alone and after treatment with 1-(2fluoro-5-methyl-beta-L-arabinofuranosyl)-uracil (L-FMAU) breaks humoral and cell-mediated immune tolerance in chronic woodchuck hepatitis virus infection. J Virol 76(11):5305-5314

46. Walter E, Keist R, Niederost B et al (1996) Hepatitis B virus infection of tupaia hepatocytes in vitro and in vivo. Hepatology 24(1):1-5 
47. Washburn ML, Bility MT, Zhang L et al (2011) A humanized mouse model to study hepatitis $\mathrm{C}$ virus infection, immune response, and liver disease. Gastroenterology 140(4):1334-1344

48. Zhang DY, Friedman SL (2012) Fibrosis-dependent mechanisms of hepatocarcinogenesis. Hepatology 56(2):769-775

49. Ghiassi-Nejad Z, Hernandez-Gea V, Woodrell C et al (2012) Reduced hepatic stellate cell expression of KLF6 tumor suppressor isoforms amplifies fibrosis during acute and chronic rodent liver injury. Hepatology. doi:10.1002/hep.26056. [Epub ahead of print].

50. Kodama $T$, Takehara $T$, Hikita $H$ et al (2011) Increases in p53 expression induce CTGF synthesis by mouse and human hepatocytes and result in liver fibrosis in mice. J Clin Investig 121(8): 3343-3356

51. Roderburg C, Urban GW, Bettermann K et al (2011) Micro-RNA profiling reveals a role for miR-29 in human and murine liver fibrosis. Hepatology 53(1):209-218

52. Cushing L, Kuang PP, Qian J et al (2011) miR-29 is a major regulator of genes associated with pulmonary fibrosis. Am J Respir Cell Mol Biol 45(2):287-294

53. Kogure T, Costinean S, Yan I et al (2012) Hepatic miR-29ab1 expression modulates chronic hepatic injury. J Cell Mol Med 16(11):2647-2654

54. Seki E, De Minicis S, Osterreicher $\mathrm{CH}$ et al (2007) TLR4 enhances TGF-beta signaling and hepatic fibrosis. Nat Med 13(11):1324-1332

55. Friedman SL, Bansal MB (2006) Reversal of hepatic fibrosis: fact or fantasy? Hepatology 43(2 Suppl 1):S82-S88

56. Ogawa T, Enomoto M, Fujii H et al (2012) MicroRNA-221/222 upregulation indicates the activation of stellate cells and the progression of liver fibrosis. Gut 61(11):1600-1609

57. Galardi S, Mercatelli N, Farace MG, Ciafre SA (2011) NF-kB and c-Jun induce the expression of the oncogenic miR-221 and miR-222 in prostate carcinoma and glioblastoma cells. Nucleic Acids Res 39(9):3892-3902

58. Lee KS, Buck M, Houglum K, Chojkier M (1995) Activation of hepatic stellate cells by TGF alpha and collagen type I is mediated by oxidative stress through c-myb expression. J Clin Investig 96(5):2461-2468

59. Lang A, Schoonhoven R, Tuvia S et al (2000) Nuclear factor kappaB in proliferation, activation, and apoptosis in rat hepatic stellate cells. J Hepatol 33(1):49-58

60. Kutay H, Bai S, Datta J et al (2006) Downregulation of miR-122 in the rodent and human hepatocellular carcinomas. J Cell Biochem 99(3):671-678

61. Bai S, Nasser MW, Wang B et al (2009) MicroRNA-122 inhibits tumorigenic properties of hepatocellular carcinoma cells and sensitizes these cells to sorafenib. J Biological Chem 284(46): 32015-32027

62. Hou J, Lin L, Zhou W et al (2011) Identification of miRNomes in human liver and hepatocellular carcinoma reveals miR-199a/b-3p as therapeutic target for hepatocellular carcinoma. Cancer Cell 19(2):232-243

63. Ji J, Shi J, Budhu A et al (2009) MicroRNA expression, survival, and response to interferon in liver cancer. N Engl J Med 361(15): $1437-1447$

64. • Kota J, Chivukula RR, O’Donnell KA et al (2009) Therapeutic microRNA delivery suppresses tumorigenesis in a murine liver cancer model. Cell 137(6):1005-1017. The first study to show the viral mediated delivery of microRNA is efficient in the suppression of sponaneous liver tumor with a mouse model.

65. Meng F, Henson R, Wehbe-Janek H et al (2007) MicroRNA-21 regulates expression of the PTEN tumor suppressor gene in human hepatocellular cancer. Gastroenterology 133(2):647-658

66. Wang B, Majumder S, Nuovo G et al (2009) Role of microRNA-155 at early stages of hepatocarcinogenesis induced by choline-deficient and amino acid-defined diet in C57BL/6 mice. Hepatology 50(4): $1152-1161$

67. Wang B, Hsu SH, Majumder S et al (2010) TGFbeta-mediated upregulation of hepatic miR-181b promotes hepatocarcinogenesis by targeting TIMP3. Oncogene 29(12):1787-1797

68. Gabriely G, Wurdinger T, Kesari S et al (2008) MicroRNA 21 promotes glioma invasion by targeting matrix metalloproteinase regulators. Mol Cell Biol 28(17):5369-5380

69. Garofalo M, Di Leva G, Romano G et al (2009) miR-221\&222 regulate TRAIL resistance and enhance tumorigenicity through PTEN and TIMP3 downregulation. Cancer Cell 16(6):498-509

70. Davis ME, Chen ZG, Shin DM (2008) Nanoparticle therapeutics: an emerging treatment modality for cancer. Nat Rev Drug Discov 7(9):771-782

71. Daya S, Berns KI (2008) Gene therapy using adeno-associated virus vectors. Clin Microbiol Rev 21(4):583-593

72. Trang P, Medina PP, Wiggins JF et al (2010) Regression of murine lung tumors by the let-7 microRNA. Oncogene 29(11):1580-1587

73. Cunningham AP, Andrews LG, Tollefsbol TO (2007) Retrovirusmediated RNA interference. Targeting hTERT through stable expression of short-hairpin RNA. Methods Mol Biol 405:39-46

74. Hatziapostolou M, Polytarchou C, Aggelidou E et al (2011) An HNF4alpha-miRNA inflammatory feedback circuit regulates hepatocellular oncogenesis. Cell 147(6):1233-1247

75. Sonoke S, Ueda T, Fujiwara K et al (2011) Galactose-modified cationic liposomes as a liver-targeting delivery system for small interfering RNA. Biological Pharm Bull 34(8):1338-1342

76. Yano J, Hirabayashi K, Nakagawa S et al (2004) Antitumor activity of small interfering RNA/cationic liposome complex in mouse models of cancer. Clin Cancer Res 10(22):7721-7726

77. Zimmermann TS, Lee AC, Akinc A et al (2006) RNAi-mediated gene silencing in non-human primates. Nature 441(7089):111-114

78. - Semple SC, Akinc A, Chen J et al (2010) Rational design of cationic lipids for siRNA delivery. Nature biotechnol 28(2):172-176. This paper demonstrated the application of a newly designed SNALP in siRNA-mediated hepatic gene silencing.

79. Akinc A, Querbes W, De S et al (2010) Targeted delivery of RNAi therapeutics with endogenous and exogenous ligand-based mechanisms. Mol Ther 18(7):1357-1364

80. Chen Y, Zhu X, Zhang X et al (2010) Nanoparticles modified with tumor-targeting scFv deliver siRNA and miRNA for cancer therapy. Mol Ther 18(9):1650-1656

81. Pramanik D, Campbell NR, Karikari C et al (2011) Restitution of tumor suppressor microRNAs using a systemic nanovector inhibits pancreatic cancer growth in mice. Mol Cancer Ther 10(8):1470-1480

82. Akao Y, Nakagawa Y, Hirata I et al (2010) Role of anti-oncomirs miR-143 and -145 in human colorectal tumors. Cancer Gene Ther 17(6):398-408

83. Piao L, Zhang M, Datta J et al (2012) Lipid-based nanoparticle delivery of Pre-miR-107 inhibits the tumorigenicity of head and neck squamous cell carcinoma. Mol Ther 20(6):1261-1269

84. Wu Y, Crawford M, Yu B et al (2011) MicroRNA delivery by cationic lipoplexes for lung cancer therapy. Mol Pharm 8(4):1381-1389

85. Sioud M, Sorensen DR (2003) Cationic liposome-mediated delivery of siRNAs in adult mice. Biochem Biophys Res Commun 312(4):1220-1225

86. Liu Y, Huang L (2010) Designer lipids advance systemic siRNA delivery. Mol Ther 18(4):669-670

87. Hafez IM, Maurer N, Cullis PR (2001) On the mechanism whereby cationic lipids promote intracellular delivery of polynucleic acids. Gene Ther 8(15):1188-1196

88. Wisse E, Jacobs F, Topal B et al (2008) The size of endothelial fenestrae in human liver sinusoids: implications for hepatocytedirected gene transfer. Gene Ther 15(17):1193-1199 
89. Burnett JC, Rossi JJ, Tiemann K (2011) Current progress of siRNA/shRNA therapeutics in clinical trials. Biotechnol J 6(9): $1130-1146$

90. Park JK, Kogure T, Nuovo GJ et al (2011) miR-221 silencing blocks hepatocellular carcinoma and promotes survival. Cancer Res 71(24):7608-7616

91. Fluiter K, ten Asbroek AL, de Wissel MB et al (2003) In vivo tumor growth inhibition and biodistribution studies of locked nucleic acid (LNA) antisense oligonucleotides. Nucleic Acids Res 31(3):953-962

92. You Y, Moreira BG, Behlke MA, Owczarzy R (2006) Design of LNA probes that improve mismatch discrimination. Nucleic Acids Res 34(8):e60

93. - Lanford RE, Hildebrandt-Eriksen ES, Petri A et al (2010) Therapeutic silencing of microRNA-122 in primates with chronic hepatitis C virus infection. Science 327(5962):198-201. First evidence to show the silencing of miR-122 by LNA modified antimir can be used to inbhit HCV infection in primates.

94. Ghosh SS, Takahashi M, Thummala NR et al (2000) Liverdirected gene therapy: promises, problems and prospects at the turn of the century. J Hepatol 32(1 Suppl):238-252
95. Jin X, Yang YD, Li YM (2008) Gene therapy: regulations, ethics and its practicalities in liver disease. World $\mathrm{J}$ Gastroenterol 14(15):2303-2307

96. Anderson WF (1998) Human gene therapy. Nature 392(6679 Suppl):25-30

97. Coulouarn C, Factor VM, Andersen JB et al (2009) Loss of miR122 expression in liver cancer correlates with suppression of the hepatic phenotype and gain of metastatic properties. Oncogene 28(40):3526-3536

98. • Trajkovski M, Hausser J, Soutschek J et al (2011) MicroRNAs 103 and 107 regulate insulin sensitivity. Nature 474(7353):649-653. First study to show the therapeutic potential of antagomir mediated miR-103/107 reduction that improved glucose homeostasis and insulin sensitivity.

99. Castoldi M, Vujic Spasic M, Altamura S et al (2011) The liverspecific microRNA miR-122 controls systemic iron homeostasis in mice. J Clin Investig 121(4):1386-1396 